\title{
Implementing K-Star Algorithm to Monitor Tyre Pressure using Extracted Statistical Features from Vertical Wheel Hub Vibrations
}

\author{
P. S. Anoop", V. Sugumaran and Hemanth Mithun Praveen \\ SMBS, VIT University Chennai campus, Chennai - 600127, Tamil Nadu, India; \\ anoopps.vit@gmail.com, v_sugu@yahoo.com, hemanth.mithun.praveen@gmail.com
}

\begin{abstract}
Objectives: Tyre pressure monitoring systems are automotive electronic systems used to monitor the automobile tyre pressure. The existing systems use pressure sensors or wheel speed sensors. They depend on batteries and radio transmitters which would add up to cost and complexity. Methods/Analysis: This paper proposes a new machine learning approach to monitor the tyre pressure. Vertical vibrations are extracted from a wheel hub of a moving vehicle using an accelerometer and are classified using machine learning techniques. The statistical features are extracted from the vibration signal and the features are classified using K Star algorithm. Findings: A reasonably high classification accuracy of $89.16 \%$ was obtained. Application/Improvements: The proposed model can be used for monitoring the automobile tyre pressure successfully.
\end{abstract}

Keywords: Automobile, K Star Algorithm, Machine Learning, Statistical Features, Tyre Pressure Monitoring System

\section{Introduction}

Tyres can be classified as pneumatic and non-pneumatic tyres depending on weather they use air to maintain their profile or not. Non-pneumatic tyres do not use air but thick polymer to maintain their shape. They are used where pneumatic tyres could fail such as demolition sites. Pneumatic tyres are commonly used in automobiles as they provide a comfortable ride; the pressurized gas within the tyre acts as a spring and dampens most of the vibration. Keeping in mind, the different levels of stability, comfort and fuel economy tyres come in different construction types; diagonal, radial and bias belt construction. A code imprinted on the sidewalls of the tyre is used to understand the tyres specification. The tyre chosen for this study was coded as '145/70 R12 69 T'. Here the width of the tyre is 145 millimeters; the side wall height is $70 \%$ that of the width and the tyre construction type is a radial tyre. This is denoted by the letter ' $R$ '. The rim size required for the tyre is 12 inches. ' 69 ' is the load index value. It denotes the maximum load allowed. Here ' 69 ' correspond to $325 \mathrm{~kg}$. ' $\mathrm{T}$ ' is the speed rating code and denotes the maximum safe speed of the tyre. Here ' $T$ ' corresponds to $190 \mathrm{~km} / \mathrm{hr}$. A radial tyre was chosen for the study Figure 1 as they are commonly used in automobiles due to their contribution to fuel economy and comfort. Tyre pressure monitoring systems or TPMS for short are active automotive electronics which monitor the tyre pressure of an automobile. TPMS can monitor individual tyres and report the tyre pressure to the driver. Based on the mode of operation TPMS may be classified as direct systems; which use barometric pressure sensors to read the air pressure inside the tyre or indirect systems; which rely on external factors outside the tyre such as wheel radius. A tyre must be inflated to the vehicle manufactures specification. This will ensure proper handling and fuel economy. Direct tyre pressure monitoring systems depend on integrated barometric pressure sensors and temperature sensors ${ }^{1}$. The temperature is measured so as to correct the errors generated by the barometric pressure

${ }^{*}$ Author for correspondence 
sensor at higher temperatures. These systems can measure tyre pressure in both static and dynamic conditions. Every TPMS sensor is equipped a controller, barometric pressure sensor, wakeup circuitry, transmitter and power source 2 . However, in most cases replacing a battery requires separation of the tyre from the rim. Moreover some systems are provided with an integrated battery which does not allow replacement. All each wheel will require a dedicated sensor which will add up the cost and RF pollution when additional wheels are used for snow tyres or off-road tyres.

With limited power available direct TPMS system depends on 'wake up' circuit. This circuit triggers the sensor and transmitter to resume from standby mode. Some systems depend on an accelerometer to detect the centrifugal acceleration caused by the movement of the tyre from rest. This sends an activation signal to the sensor ${ }^{3}$. Other systems depend on an activation signal from the main TPMS control unit through a low frequency receiver. The sensor is enclosed within a polymer case which may then be strapped using steel bands to the rim. Some sensors can be positioned at the neck of the tyre. A common defect reported by direct TPMS users was that the valve stem would corrode as the stem and the rim are of different metals and would break with the slightest amount of torque. Moreover self-sealing solvents could interfere with sensor operation as it would clog up the sensor port. The sensor will remain non-operational until cleaning. Indirect tyre pressure monitoring systems depend on the wheel speed signals from the wheel speed sensor ${ }^{4}$. When a tyre deflates its angular velocity increases slightly as the radius decreases when compared to other tyres. These wheel speeds can be detected with the wheel speed sensor used by the antilock braking system. Based on the algorithm implemented all speeds are compared. If a pressure difference occurs it will trigger a mismatch as the wheel speeds would be uneven. Indirect TPMS systems are relative in nature hence they must be reset once the tyres are pressurized to its optimum pressure. This has to be done to 'Re-learn' the required parameters. The 'Re-learning' procedure can take 5 to 60 minutes of driving. Indirect TPMS requires the vehicle to be in motion to operate ${ }^{5}$. A remote tyre pressure monitoring technique was proposed by in ${ }^{6}$. The accelerometer was connected to a signal analyzer and fixed on the tyre and the tyre was then struck with a hammer. The vibration frequency peaks generated by the tyre would alter based on the air pressure. A vibration monitoring method to measure damper condition and wheel balance was proposed by $\mathrm{in}^{\mathrm{z}}$. These parameters would be monitored when the vehicle is driven in a normal way. It was reported that the tyre pressure could also be predicated but only up to an accuracy of $12 \%$ and concluded that further research was needed to improve the classification accuracy. In $\underline{8}$ other work the information sensor fusion was better than the Bayesian method after carrying out a research on algorithms for tyre pressure monitoring based on multiple sensor information fusion and Bayesian. In Piezoelectric vibration harvesting system was proposed. The system uses a sensor to harvest vibration from a running tyre to generate electrical power, there by powering the tyre pressure monitoring sensor. A research on the influence of vehicle tyre pressure on suspension system was carried out and the vertical vibrations generated by the system were used for data analysis by applying the time - frequency approach ${ }^{10}$. $\operatorname{In}^{11}$ used the strain information acquired from a tyre to investigate tyre grip identification based on strain information where the tyre road grip condition could be estimated from the strain experienced. $\mathrm{In}^{12}$ conducted a study in which low frequency statistical estimations of rolling noise of tyre and road were calculated numerically. $\operatorname{In}^{\underline{13}}$ a survey conducted by the UK tyre industry council, between the years of 2003 to 2005 it was reported that about $50 \%$ of light vehicle crashes were due to defective tyres. The contact path to the ground increases as a tyre deflates ${ }^{14}$. This could add rolling resistance. Extreme under-inflation will cause the tyre to heat up and destroy itself $\mathrm{f}^{15}$. Tyre wear and a drop in fuel efficiency are experienced when driving with under-inflation tyres. Tyres lose air overtime due to natural diffusion; even a new, properly mounted tyre can lose pressure up to 1 psi a month $\frac{16}{}$. Improved fuel economy, extended tyre life, decreased downtime and maintenance and improved handling can be achieved if a tyre is maintained at optimum pressure. After the literature survey conducted it could be concluded that a machine learning technique was not thoroughly investigated. Hence this paper proposes a new innovative method to monitor tyre pressure by utilizing the machine learning approach. The vertical vibrations are extracted from a wheel hub of a moving vehicle using a MEMS (Micro Electro Mechanical Systems) accelerometer and are classified using machine learning techniques. This paper reports the study conducted where the statistical features were extracted from the vibration signal and the features were classified using support vector machine algorithm. A reasonably high classification accuracy of $90 \%$ was obtained. 


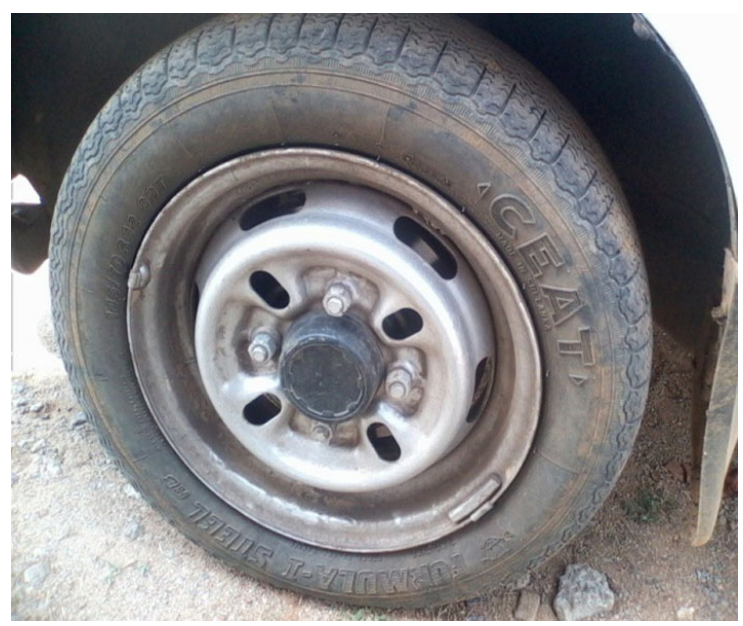

Figure 1. Tyre used for experiments.

\section{Experimental Setup}

The experiment used a front wheel drive vehicle. The tyre was of radial construction. The tyre was pressurized to $28 \mathrm{psi}$ as per vehicle specification. This was taken as normal and pressure readings of 22 psi and below was taken as puncture. The vehicle was first driven with its tyre properly inflated; the vibration signals were recorded. The tyre was then de-pressurized to 22 psi to simulate a puncture and the vehicle was driven; the vibration signals were acquired. The test speeds were limited from $20 \mathrm{~km} / \mathrm{hr}$ to $80 \mathrm{~km} / \mathrm{hr}$. The vehicle was driven in a normal manner. A normal driving method ensured that the speeds were varied rather than fixed values. Speeds below $20 \mathrm{~km} / \mathrm{hr}$ did not yield sufficient amplitude hence they were classified as idle irrespective of their tyre state. A triaxial MEMS accelerometer was used to acquire the data. Figure 2 portraits the accelerometer module as a whole. Figure 3 illustrates the axis of the accelerometer. Figure 4 shows the experimental setup. A total of 360 samples of 1000 data points each at a sampling rate of $66 \mathrm{~Hz}$ were taken. To ensure that the experiment remained unbiased, 120 samples were acquired for each of the classes. The classes were titled 'Normal', 'Puncture' and 'Idle'. A data acquisition program written in visual $\mathrm{C}++$ and was used to log the incoming data through serial port. In the recent times, piezo resistive accelerometers have been extensively explored with the primary focus on the optimization of its sensitivity and measurement bandwidth. $\operatorname{In}^{17}$ systematic analysis has been carried to optimize the sensitivity. The MEMS accelerometer module used was water proofed and fixed to the rear right wheel hub of the car Figure 4 by means of an adhesive. A shielded wire was used to minimize external electronic interference as it could generate errors during sampling. The details of the accelerometer are tabulated in Table 1. The specifications of the DAQ used are tabulated in Table 2. The sample waveform of the tyre's vibration from the MEMS sensor for the conditions of normal, puncture and idle are shown in Figure 5 to Figure 7 respectively.

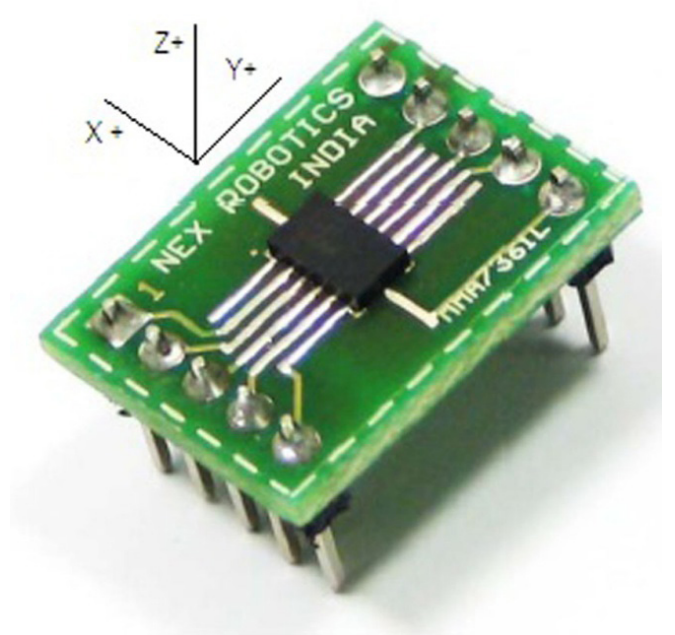

Figure 2. MEMS accelerometer module.

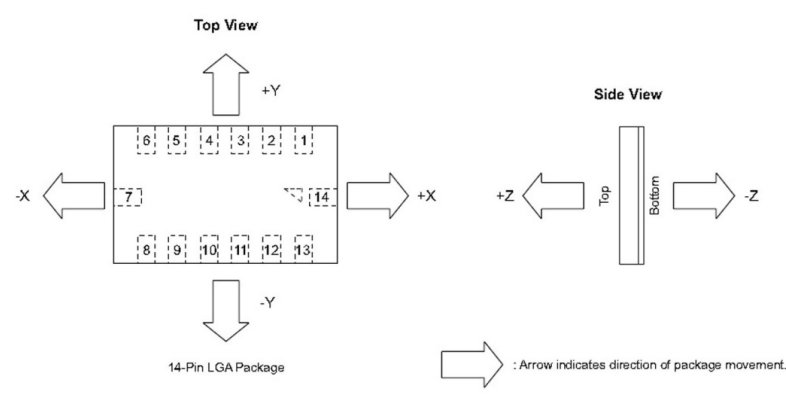

Figure 3. MEMS accelerometer axis (from datasheet).

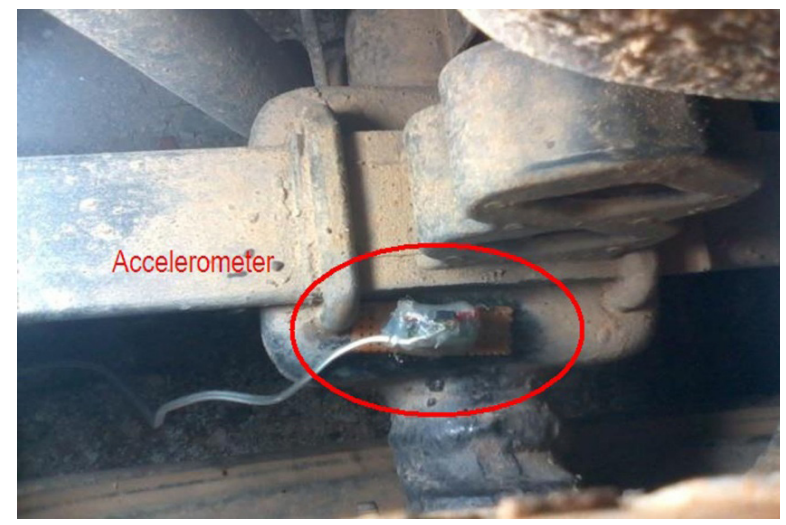

Figure 4. MEMS accelerometer fixed on the axel. 


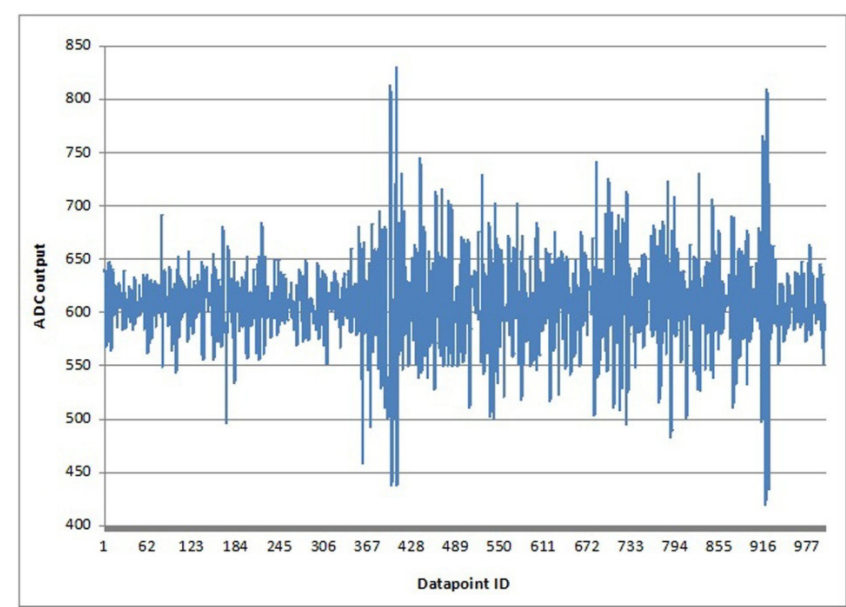

Figure 5. Sample signal of normal condition from MEMS accelerometer.

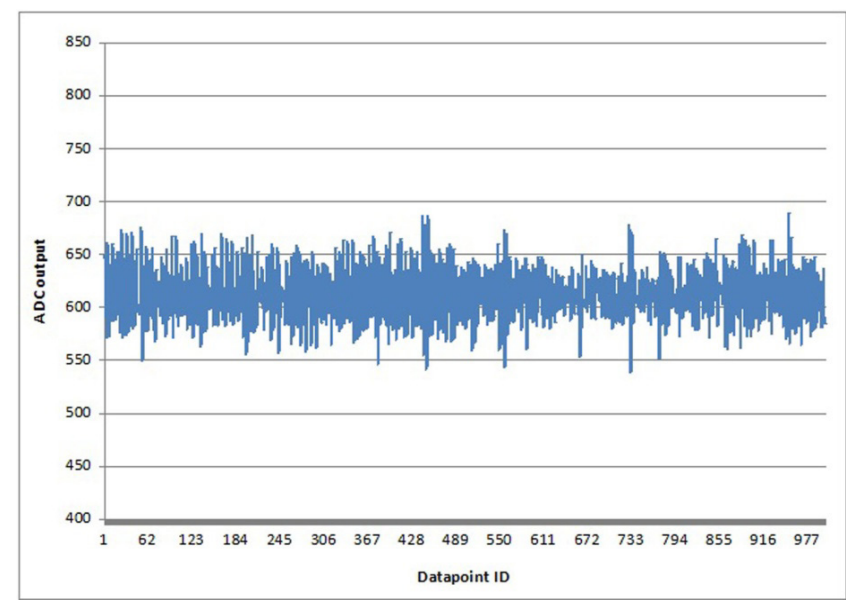

Figure 6. Sample signal of puncture condition from MEMS accelerometer.

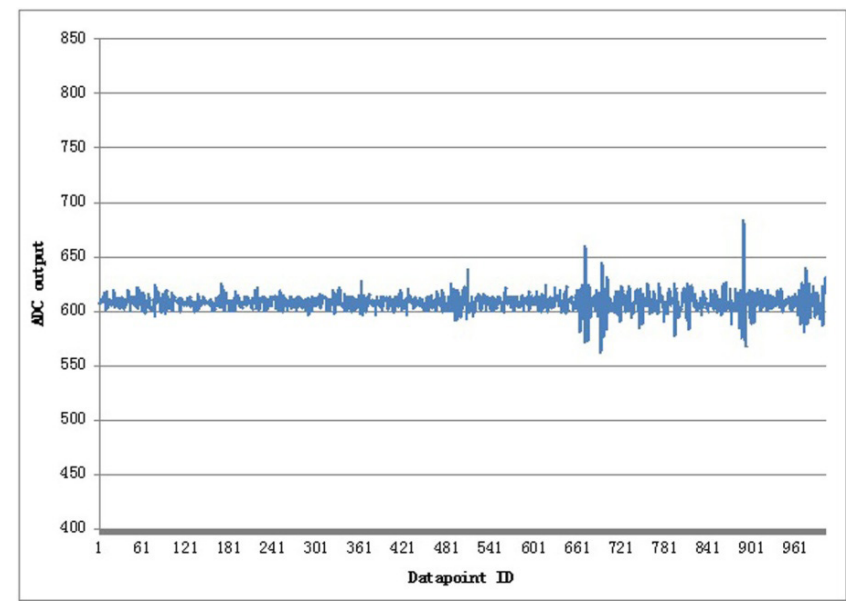

Figure 7. Sample signal of idle condition from MEMS accelerometer.
Table 1. MEMS accelerometer specification

\begin{tabular}{|l|l|}
\hline FEATURES & SPECIFICATION \\
\hline Make & $\begin{array}{l}\text { Freescale Semiconductor } \\
\text { (MMA7361L) }\end{array}$ \\
\hline Weight & $\begin{array}{l}<1 \mathrm{~g} \text { (accelerometer only) } 5 \mathrm{~g} \text { with } \\
\text { supporting electronics }\end{array}$ \\
\hline Type & MEMS \\
\hline Number of Axis & 3 \\
\hline Description & $\pm 1.5 \mathrm{~g}$ \\
$\pm 6 \mathrm{~g}$ \\
Selectable range
\end{tabular}

Table 2. Specification of DAQ system used for MEMS sensor

\begin{tabular}{|l|l|}
\hline FEATURES & SPECIFICATION \\
\hline Make & Self \\
\hline PC communication & USB/RS232 \\
\hline Number of input Channel & 3 \\
\hline ADC Type & Successive approximation \\
\hline ADC resolution & $10 \mathrm{bit}$ \\
\hline Max sampling rate & $15 \mathrm{kHz}$ \\
\hline
\end{tabular}

\subsection{Sampling Rate Calculation}

The tyre used was $145 / 70$ radial tyre with a max pressure rating of $44 \mathrm{psi}$.

- Radius of tyre (R) $=25 \mathrm{~cm}$.

- Maximum Speed in $\mathrm{km} / \mathrm{hr}(\mathrm{S})=80 \mathrm{~km} / \mathrm{hr}(22.22$ $\mathrm{m} / \mathrm{s})$.

- Minimum Speed in $\mathrm{km} / \mathrm{hr}(\mathrm{S})=20 \mathrm{~km} / \mathrm{hr}(5.55$ $\mathrm{m} / \mathrm{s})$.

Since the wheel radius and the speed in known speed can be achieved by equating it to known variables (R, $S$ ).

Minimum Speed and Frequency.

- $2 \Pi \times 0.25(\mathrm{R}) \times$ Speed $/ 60=5.55$.

- Speed $=212 \mathrm{rpm}$. 
- $\quad$ Frequency $=212 / 60=3.53 \mathrm{~Hz}$.

Maximum Speed and Frequency.

- $2 \Pi \times 0.25(\mathrm{R}) \times$ Speed $/ 60=22.22$.

- Speed $=848$.

- Frequency $=848 / 60=14.13 \mathrm{~Hz}$.

Based on Nyquist Shannon sampling theorem the minimum sampling rate should be at least double the highest incoming frequency to avoid antialiasing $\frac{18}{}$. The minimum sampling rate required is $28.26 \mathrm{~Hz}$ and hence the sampling rate was set at $66 \mathrm{~Hz}$.

\section{Machine Learning}

Machine learning consist of three phases; Feature extraction, feature selection, feature classification. The vibration signal will be used to extract the statistical features to yield the required parameters.

\subsection{Feature Extraction}

In feature extraction the statistical parameters such as 'mean', 'mode' etc. are extracted from the vibration signal. Spread sheet software was used to complete the task.

\subsection{Feature Selection}

In the feature selection the best contributing features would be selected by the algorithm. The remaining features would be removed to reduce the computational load and to improve classification accuracy. Figure 8 shows the decision tree generated by the J48 algorithm. Table 3 shows the detailed accuracy by class for the untrained J48 classifier. Table 4 shows the stratified cross-validation details for the untrained J48 classifier. Table 5 shows the confusion matrix generated by the untrained J48 classifier. Table 6 shows the parameters for the J48 classifier.

\section{Number of Leaves: $\quad 16$ \\ Size of the Tree: $\quad 31$}

Time taken to build model: 0.01 seconds

From the feature selection process, the features selected were standard error, mean, skewness, kurtosis and median. The remaining attributes were rejected. 'Minimum' and 'range' were rejected even though selected by the classifier. It was found that the classification accuracy would drop (by 1\%) if used and would also increase the computational load. Classification accuracy of $88.78 \%$ was attained for the untrained J48 classifier. 102/120 sam- ples were correctly classified as normal, 103/120 samples were correctly classified as puncture and 120/120 samples were correctly classified as idle. These could be noted as the diagonal elements in the confusion matrix Table 5. This denotes the correctly classified classes. However, $17 / 120$ samples that is $14.16 \%$ of the class 'puncture' was incorrectly classified as normal. Moreover 16/120 samples that is $13.33 \%$ of the class 'Normal' were incorrectly classified as puncture. Adding to the previous misclassification two samples of normal were misclassified as idle about $1.81 \%$. This indicated that a study is required for reducing the misclassification thereby increasing the classification accuracy.

Table 3. Detailed accuracy by class for J48

\begin{tabular}{|l|l|l|l|l|l|l|}
\hline TP & FP & PR & R & F & RO & C \\
\hline 0.858 & 0.067 & 0.866 & 0.858 & 0.862 & 0.919 & N \\
\hline 1 & 0.008 & 0.984 & 1 & 0.992 & 0.993 & P \\
\hline 0.85 & 0.071 & 0.857 & 0.85 & 0.854 & 0.889 & I \\
\hline 0.903 & 0.049 & 0.902 & 0.903 & 0.902 & 0.933 & W \\
\hline
\end{tabular}

Table 4. Cross-validation for J48

\begin{tabular}{|l|l|}
\hline Parameter & Value/ Action \\
\hline Correctly Classified Instances & $\mathbf{3 2 5 / 3 6 0}$ \\
\hline Kappa statistic & 0.8542 \\
\hline Root mean squared error & 0.2463 \\
\hline Root relative squared error & $52.2453 \%$ \\
\hline Incorrectly Classified Instances & $35 / 360$ \\
\hline Mean absolute error & 0.0809 \\
\hline Relative absolute error & $18.1921 \%$ \\
\hline Total Number of Instances & 360 \\
\hline
\end{tabular}

Table 5. Confusion matrix generated J48 tree

\begin{tabular}{|l|l|l|l|}
\hline Classified as & Normal & puncture & idle \\
\hline Normal & 102 & 16 & 2 \\
\hline Puncture & 17 & 103 & 0 \\
\hline Idle & 0 & 0 & 120 \\
\hline
\end{tabular}

Table 6. Values for objects of the trained J48 tree

\begin{tabular}{|l|l|l|}
\hline Sl. No. & Objects & Value \\
\hline $\mathbf{1}$ & Confidence factor & 0.25 \\
\hline $\mathbf{2}$ & Minimum number of objects & 2 \\
\hline $\mathbf{3}$ & Number of folds & 3 \\
\hline $\mathbf{4}$ & Seed & 1 \\
\hline
\end{tabular}




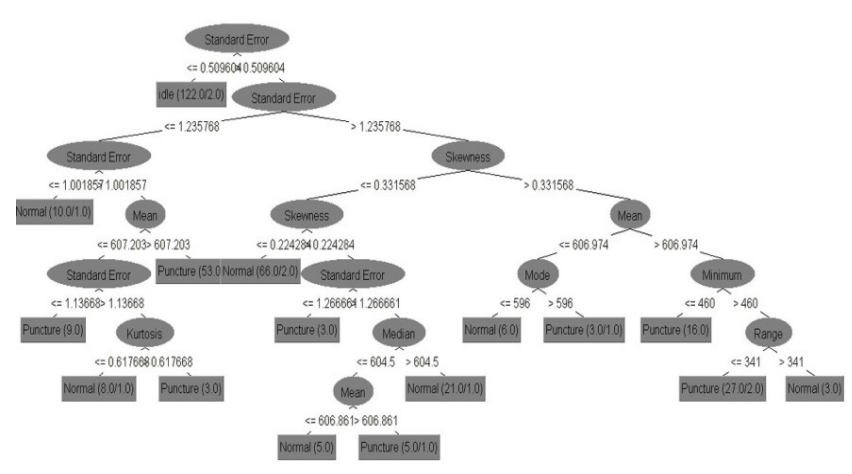

Figure 8. Decision tree generated using the J48 tree algorithm.

\subsection{Feature Classification}

Lazy classifiers are useful for datasets with few attributes. They are based on 'lazy learning method'. In lazy learning method the algorithm does not generalize the data set until a query is made, in contrast to eager learning method where the training set is first generalized. $\mathrm{K}^{\star}$ is an instance-based classifier, that is the class of a test instance is based upon the class of those training instances similar to it, as determined by some similarity function. It differs from other instance-based learners in that it uses an entropy-based distance function. The algorithm depends on two variables 'global blend' and 'missing Mode'. Missing Mode determines how missing attribute values are treated the algorithm uses four modes to treat missing attributes. The modes were as follows:

- Ignore the instances with missing values (M1).

- Normalize over the attributes (M2).

- Treat missing values as maximally different (M3).

- Average column entropy curves (M4).

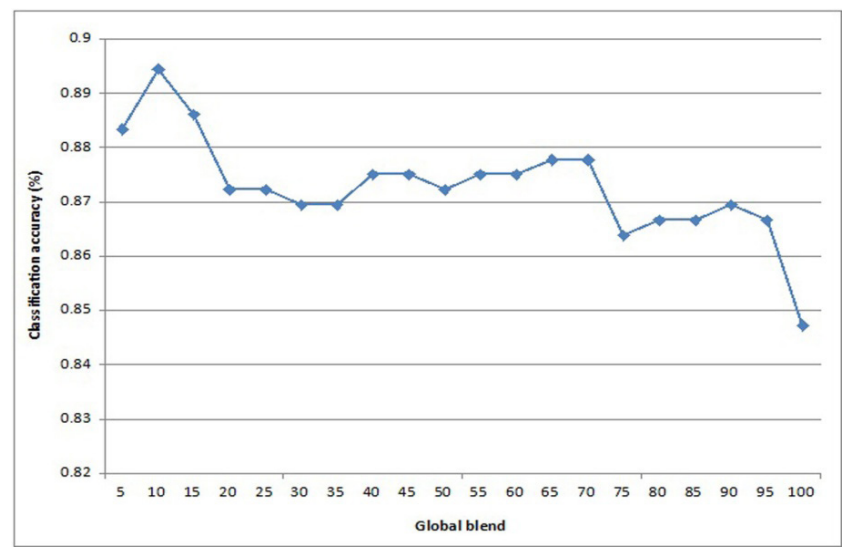

Figure 9. $M(1)$ vs. classification accuracy.

\section{Results and Discussion}

All four modes in lazy classifiers were experimented however no change was to be found in the classification accuracy hence the details for the default mode which is 'Average column entropy curves' is explained. Table 7 shows the confusion matrix generated by the trained classifier. The results $\mathrm{M}(1)$ vs. classification accuracy is displayed in Figure 9. The stratified cross-validation details and the detailed accuracy by class are given in Table 8 and Table 9 respectively. Varying the parameter titled 'global blend' from 1 to 100 in steps of ' 5 ' causes a steady drop in classification accuracy, As mentioned earlier all four modes were tested and yielded the same result. Figure 9 displays $\mathrm{M}(1)$ vs. classification accuracy. The values of objects for the trained algorithm are shown in Table 9. From the confusion matrix Table 7 it can be noted that $98 / 120$ samples were correctly classified as normal, 103/120 samples were correctly classified as puncture and 120/120 samples were correctly classified as idle. The classifier achieved a maximum classification accuracy of $89.16 \%$ after training. Values for objects of the trained $\mathrm{K}^{\star}$ classifier are shown in Table 10.

Table 7. Confusion matrix for $\mathrm{K}^{*}$

\begin{tabular}{|l|l|l|l|}
\hline Classified as & Normal & Puncture & Idle \\
\hline Normal & 98 & 17 & 5 \\
\hline Puncture & 17 & 103 & 0 \\
\hline Idle & 0 & 0 & 120 \\
\hline
\end{tabular}

Table 8. Cross-validation for $\mathrm{K}^{\star}$

\begin{tabular}{|l|l|}
\hline Parameters & Results \\
\hline Correctly Classified Instances & $321 / 360$ \\
\hline Kappa statistic & 0.8375 \\
\hline Root mean squared error & 0.2395 \\
\hline Root relative squared error & $50.80 \%$ \\
\hline Incorrectly Classified Instances & $39 / 360$ \\
\hline Mean absolute error & 0.0897 \\
\hline Relative absolute error & $20.17 \%$ \\
\hline Total Number of Instances & 360 \\
\hline
\end{tabular}

Table 9. Detailed accuracy by class for $\mathrm{K}^{*}$

\begin{tabular}{|l|l|l|l|l|l|l|}
\hline TP & FP & PR & R & F & RO & C \\
\hline 0.817 & 0.071 & 0.852 & 0.852 & 0.817 & 0.947 & N \\
\hline 0.9 & 0.071 & 0.858 & 0.858 & 0.858 & 0.965 & P \\
\hline
\end{tabular}




\begin{tabular}{|l|l|l|l|l|l|l|}
\hline 1 & 0.021 & 0.96 & 1 & 0.98 & 1 & $\mathrm{I}$ \\
\hline 0.892 & 0.054 & 0.89 & 0.892 & 0.891 & 0.971 & W \\
\hline
\end{tabular}

Table 10. Values for objects of the trained $\mathrm{K}^{\star}$ classifier

\begin{tabular}{|l|l|l|}
\hline Sl no. & Objects & Value \\
\hline $\mathbf{1}$ & Global blend & 10 \\
\hline $\mathbf{2}$ & Missing mode & $\mathrm{M}(4)$ \\
\hline
\end{tabular}

\section{Conclusion}

A new method which uses a machine learning approach for automobile tyre pressure monitoring was proposed. The statistical features were extracted from the vibration signals acquired from a wheel hub of a moving vehicle. The feature selection process was carried out using the J48 classifier. K Star algorithms were used to classify the signal. A maximum classification accuracy of $89.16 \%$ was obtained. The accuracy is good enough for many practical applications.

\section{Reference}

1. Velupillai S, Guvenc L. Tyre Pressure Monitoring [Applications of Control]. IEEE Control Systems. 2007 Dec; 27(6):22-5.

2. Tyre tread depth and wet weather stopping distances. 2015. Available from: http://www.rospa.com/road-safety/advice/ vehicles/tyre-safety-technology/pressure-monitoring-systems

3. Wei C, Zhou W, Wang Q, Xia X, Li X. TPMS (Tyre-Pressure Monitoring System) sensors: Monolithic integration of surface-micro machined piezo resistive pressure sensor and self-testable accelerometer. Microelectronic Engineering. 2012 Mar; 91(1):167-73.

4. NIRA Dynamics $A B$ and Dunlop Tech GmbH. Indirect Tyre Pressure Monitoring Systems - Myths and Facts. 2012. Available from: http://www.businesswire.com/news/ home/20120720005231/en/Nira-Dynamics-Indirect-TirePressure-Monitoring-Systems

5. NIRA Dynamics. 2016. Available from: http://www.niradynamics.se/

6. Remote tyre pressure sensing technique. 2013. Available from: https://archive.org/details/nasa_techdoc_19940011411
7. Craighead IA. Sensing tyre pressure, damper condition and wheel balance from vibration measurements. Proceeding of Institution of Mechanical Engineers. Part D: Journal of Automobile Engineering. 1997 Apr; 211(4):257-65.

8. Yulan Z, Yanhong Z, Yahong L. Based on multi-sensor information fusion algorithm of TPMS research. International Conference on Solid State Devices and Materials Science. Physics Procedia. 2012 Apr; 25:786-92.

9. Singh KB, Bedekar V, Taheri S, Priya S. Piezoelectric vibration energy harvesting system with an adaptive frequency tuning mechanism for intelligent tires. Mechatronics. 2012 Oct; 22(1):970-88.

10. Hamed M, Tesfa B, Aliwan M, Li G, Gu F, Ball AD. The influence of vehicle tyres pressure on the suspension system response by applying the time-frequency approach. International Conference on Automation and Computing; Brunel University, London. 2013 Sep. p. 1-6.

11. Carcaterran A, Roveri N. Tyre grip identification based on strain information: Theory and simulations. Mechanical Systems and Signal Processing. 2013 Dec; 41(1-2):564-80.

12. Dubois G, Cesbron J, Yin HP, Anfosso-Ledee F, Duhamel D. Statistical estimation of low frequency tyre/road noise from numerical contact forces. Applied Acoustics. 2013; 74(9):1085-93.

13. The role of tyre pressure in vehicle safety, injury and environment. Road safety solutions. 2007. Available from: http://www.inawise.com/liveimages/tyre-pressure-reportfinal.pdf

14. Mohsenimanesh A, Ward SM, Gilchrist MD. Stress analysis of a multi-laminated tractor tyre using non-linear $3 \mathrm{D}$ finite element analysis. Materials and Design. 2009 Apr; 30(4):1124-32.

15. Persson N. Estimation properties of a tyre pressure monitoring system. Link Opings University, SE-581 83 Link oping; 2002 Nov. p. 1-6.

16. Pearce JM, Hanlon JT. Energy conservation from systematic tyre pressure regulation. Energy Policy. 2007 Apr; 35(4):2673-7.

17. Sri RN, Vetrivel S, Mathew R, Sankar AR. Effect of electroplated gold film on the performance of a piezoresistive accelerometer with stress concentrated tiny beams. Indian Journal of Science and Technology. 2015 Aug; 8(19):1-5.

18. McLean RF, Alsop SH, Fleming JS. Nyquist - Overcoming the limitations. Journal of Sound and Vibration. 2005 Feb; 280(1-2):1-20. 